\title{
Discrepancies between cerebral perfusion and metabolism after subarachnoid haemorrhage: a magnetic resonance approach
}

Jeremy Rowe, Andrew M Blamire, Zayne Domingo, Virginia Moody, Andrew Molyneux, James Byrne, Thomas Cadoux-Hudson, George Radda
MRC Magnetic

Resonance

Spectroscopy Unit, John Radcliffe

Hospital, Oxford, UK

J Rowe

A M Blamire

$Z$ Domingo

T Cadoux-Hudson

G Radda

Department of

Neurosurgery

J Rowe

T Cadoux-Hudson

Department of

Neuroradiology,

Radcliffe Infirmary,

Oxford, UK

V Moody

A Molyneux

J Byrne

Correspondence to:

Dr Jeremy Rowe,

Department of

Neurosurgery, Radcliffe

Infirmary, Woodstock Road,

Oxford OX2 6HE, UK.

Telephone 00441865

311188; fax 00441865

224898.

Received 11 December 1996 and in revised form 11 June 1997

Accepted 7 July 1997

\begin{abstract}
Introduction-There is a variable relation between angiographic vasospasm and delayed ischaemic neurological deficit (DIND). Magnetic resonance (MR) techniques have the potential to investigate the haemodynamic, metabolic, and structural changes occurring with these complications. These techniques have been applied to study DIND in patients recovering from subarachnoid haemorrhage.

Methods-Fifteen studies were performed on 11 patients, 10 with DIND. Vasospasm was diagnosed angiographically or with transcranial Doppler. The MR protocol consisted of $T 2$ weighted imaging, contrast enhanced dynamic perfusion scanning, TI weighted imaging, and two dimensional localised proton spectroscopy. Relative cerebral blood volume maps were generated from perfusion scans. Metabolite ratios were calculated from proton spectra.

Results-All patients had cortical oedema on $T 2$ weighted images, significantly more pronounced in patients of poor clinical grade $(p<0.01)$. Spectra were normal in good grade patients. Lactate was increased and $\mathbf{N}$-acetyl aspartate decreased in the poor grades, significantly worse in grade 4 compared with grade 3 patients $(p<0.05)$. Spectral changes also correlated with the severity of oedema $(p<0.05)$. Relative blood volumes were significantly higher in oedematous regions of poor compared with good grade patients $(p<0.05)$. Lactate was seen in regions of the brain with increased relative blood volume.

Conclusions-Despite the paramagnetic effects of haemorrhage, or of the coils and clips used to treat aneurysms, this study demonstrates that patients recovering from subarachnoid haemorrhage can undergo complex MR studies. Oedema, lactate, and increased relative blood volume correlate well with each other and with DIND and poor clinical grade.
\end{abstract}

(F Neurol Neurosurg Psychiatry 1998;64:98-103)

Keywords: subarachnoid haemorrhage; vasospasm; magnetic resonance; delayed ischaemic neurological deficit

Vasospasm, which is associated with delayed ischaemic neurological deficits (DIND), is regarded as the leading cause of death and dis- ability in patients surviving the initial ictus of subarachnoid haemorrhage. ${ }^{1}$ The relation, however, of angiographic vasospasm with DIND is variable, vasospasm sometimes being found in asymptomatic patients. ${ }^{2}$ Cerebral blood flow has been studied using ${ }^{133} \mathrm{Xe}$, positron emission tomography (PET), and single photon emission tomography (SPECT) in patients with vasospasm after subarachnoid haemorrhage. ${ }^{3}$ Whereas most of these studies have shown that cerebral blood flow is reduced during the period of angiographic vasospasm, these results have also been variable. ${ }^{3}$ In addition raised cerebral blood volumes have been reported in poor grade patients, suggesting a compensatory dilatation of the capillary bed in response to the proximal vasospasm. ${ }^{4}$ PET studies have also suggested the existence of an additional toxic or metabolic abnormality as the reduction in oxygen consumption is not explained by the decrease in blood flow. ${ }^{5-9}$

In an attempt to explore the pathophysiology of DIND, an integrated magnetic resonance (MR) protocol was used to examine the structural, metabolic, and haemodynamic changes which occur. In addition, given concerns that haemorrhage, and the coils and clips used to treat aneurysms, could have paramagnetic effects, degrading the quality of the MR data, the feasability of acquiring this information in a selected neurologically stable group of patients recovering from subarachnoid haemorrhage was assessed, this work being undertaken as a pilot study.

\section{Clinical material and methods}

PATIENTS

Fifteen studies were performed on 11 selected patients with aneurysmal subarachnoid haemorrhage (table 1). Their mean age was 49 (range 31-63) years, and there were 10 women and one man in this group. Patients were selected because they had DIND but were stable, or in one case because of the risk of developing DIND due to extensive subarachnoid blood. The mean time of first study was day 11 , range 3-26 (day 0 being the day of the presenting haemorrhage). The range reflects constraints of the patients' clinical stability and the availability of scanning time. All patients were assessed neurologically by a neurosurgeon and graded according to the Hunt and Hess scale ${ }^{10}$ on the day of the MR study. The initial diagnostic CT was used by a neurosurgeon to grade the extent of subarachnoid blood, and reflecting the selection of patients with DIND, 
Table 1 Patient details (clinical assessment used the Hunt and Hess grading system; Glasgow coma scores (GCS) were not assigned when focal neurology was causing verbal impairment)

\begin{tabular}{|c|c|c|c|c|c|c|c|c|c|}
\hline \multirow{2}{*}{$\begin{array}{l}\text { Case } \\
\text { No }\end{array}$} & \multirow[b]{2}{*}{ Sex } & \multirow[b]{2}{*}{ Age } & \multirow{2}{*}{$\begin{array}{l}\text { Fisher } \\
\text { grade }\end{array}$} & \multirow[b]{2}{*}{ Aneurysm site } & \multirow[b]{2}{*}{ Treatment } & \multirow{2}{*}{$\begin{array}{l}\text { Timing of } \\
\text { MR study }\end{array}$} & \multicolumn{3}{|c|}{ Symptoms at time of MR study } \\
\hline & & & & & & & Grade & GCS & Deficit \\
\hline 1 & $M$ & 31 & 3 & ACoA & Clipped day 1 & Day 10 & 2 & 13 & Confused: no focal signs \\
\hline 2 & $\mathrm{~F}$ & 49 & 3 & $\mathrm{ACoA}$ & Clipped day 1 & Day 3 & 1 & 15 & None \\
\hline \multirow[t]{2}{*}{3} & $\mathrm{~F}$ & 58 & 4 & Right MCA & Embolised day 10 & Day 13 & 4 & 12 & left hemiplegia \\
\hline & & & & & & Day 19 & 3 & 15 & Improving left hemiparesis \\
\hline \multirow[t]{2}{*}{4} & $\mathrm{~F}$ & 62 & 3 & Left MCA & Embolised day 10 & Day 13 & 4 & - & Drowsy, dysphasic, right hemiplegia \\
\hline & & & & & & Day 27 & 3 & - & Alert, dysphasic, improving hemiparesis \\
\hline 5 & $\mathrm{~F}$ & 44 & 3 & Pericallosal & Clipped day 1 & Day 4 & 3 & - & Mute, left hemiplegia \\
\hline 6 & $\mathrm{~F}$ & 54 & 3 & ACoA & Embolised day 2 & Day 5 & 1 & 15 & Fully resolved right hemiparesis \\
\hline 7 & $\mathrm{~F}$ & 63 & 4 & Right ant choroidal & Not secured & Day 9 & 4 & 10 & Drowsy, left hemiplegia \\
\hline 8 & $\mathrm{~F}$ & 44 & 3 & Left ant choroidal & Embolised day 5 & Day 15 & 2 & - & $\begin{array}{l}\text { Residual expressive dysphasia, resolved right arm } \\
\text { monoparesis }\end{array}$ \\
\hline \multirow[t]{2}{*}{9} & $\mathrm{~F}$ & 33 & 2 & $\begin{array}{l}\text { Left MCA and left } \\
\text { opthalmic }\end{array}$ & Embolised day 2 & Day 12 & 3 & - & Dysphasic, dysgraphic, amnesic \\
\hline & & & & & & Day 15 & 1 & 15 & Symptoms fully resolved \\
\hline 10 & $\mathrm{~F}$ & 44 & 3 & Basilar $2^{\circ} \mathrm{AVM}$ & Embolised day 24 & Day 26 & 3 & - & $\begin{array}{l}\text { Mute, improving bilateral weakness, but residual } \\
\text { right sided signs }\end{array}$ \\
\hline \multirow[t]{2}{*}{11} & $\mathrm{~F}$ & 54 & 3 & ACoA & Embolised day 1 & Day 9 & 3 & 13 & Confused: left leg monoplegia \\
\hline & & & & & & Day 14 & 3 & 15 & Residual left leg monoparesis \\
\hline
\end{tabular}

$\mathrm{ACoA}=$ anterior communicating artery; Ant choroidal=anterior choroidal artery; $\mathrm{MCA}=$ middle cerebral artery; $\mathrm{AVM}=$ arteriovenous malformation.

10 of the 11 patients were Fisher grade 3 or $4 .{ }^{11}$ Patients developing DIND all routinely had CT to exclude further haemorrhage or hydrocephalus as a cause for their deterioration. The diagnosis and distribution of vasospasm was assessed independently and blindly by consultant neuroradiologists from angiograms obtained for clinical reasons. Transcranial Doppler was not used in all patients reflecting consultant preference, but when applied a velocity exceeding $120 \mathrm{~cm} / \mathrm{s}$ was taken as diagnostic for middle cerebral artery and $150 \mathrm{~cm} / \mathrm{s}$ for anterior cerebral artery vasospasm. ${ }^{3}$ With one exception, all patients were studied after the aneurysm had been secured. In seven patients this was by an endovascular technique using Guglielmi detachable coils, and in three patients by conventional surgery. All patients received nimodipine and once the aneurysm had been secured, symptomatic patients were treated with volume expansion and inotropes. Informed consent was obtained before doing the study and the research protocol was approved by the Central Oxford research ethics committee.

MR PROTOCOL

Studies used a $1.5 \mathrm{~T}$ Siemens Magnetom Vision MR scanner. Initially standard T2 weighted images (turbo spin echo sequence, TE $96 \mathrm{~ms}$, TR $3976 \mathrm{~ms}$ ) were acquired, and examined for areas of signal change which defined regions of interest and guided the rest of the examination. Dynamic perfusion scanning was performed with a single T2 weighted $10 \mathrm{~mm}$ thick slice placed through the region of interest, using a spin echo prepared turboFLASH sequence (TE $80 \mathrm{~ms}$, TR $8.5 \mathrm{~ms}$, flip angle $10^{\circ}$ ) to collect 80 images, with a repetition rate of 1.2 seconds. Fifteen millilitres

Table 2 Patient investigations

\begin{tabular}{|c|c|c|c|c|c|c|}
\hline \multirow[b]{2}{*}{ Case No } & \multicolumn{2}{|l|}{ Vasospasm } & \multirow[b]{2}{*}{ T2 imaging changes } & \multirow{2}{*}{$\begin{array}{l}\text { Parenchymal } \\
\text { enhancement }\end{array}$} & \multirow[b]{2}{*}{$M R S$} & \multirow[b]{2}{*}{$C B V$} \\
\hline & Distribution & Diagnosis & & & & \\
\hline 1 & Diffuse & DSA day 9 & $\begin{array}{l}\text { Cortical, moderate, right MCA territory, } \\
\text { Deep, left caudate }\end{array}$ & $\begin{array}{l}\text { Not } \\
\text { performed }\end{array}$ & Not performed & Decreased \\
\hline 2 & None & $\begin{array}{l}\text { DSA day } 1 \\
\text { TCD day } 3\end{array}$ & $\begin{array}{l}\text { Cortical, moderate, left MCA territory, } \\
\text { Deep, left caudate }\end{array}$ & None & Not performed & Decreased \\
\hline 3 & Right MCA and ACA & DSA day 10 & $\begin{array}{l}\text { Cortical, pronounced, right } \mathrm{MCA} \\
\text { territory }\end{array}$ & None & Not performed & Increased \\
\hline 2nd study & & & Partial resolution & Moderate & Not performed & $\begin{array}{l}\text { Further } \\
\text { increase }\end{array}$ \\
\hline 4 & $\begin{array}{l}\text { Diffuse: left MCA, ACA and } \\
\text { vertebro-basilar }\end{array}$ & DSA day 10 & Cortical, pronounced, left MCA territory & None & $\begin{array}{l}\text { Increased lactate } \\
\text { decreased NAA }\end{array}$ & Increase \\
\hline 2nd study & & & Partial resolution & Pronounced & Partial recovery NAA & $\begin{array}{l}\text { Increase } \\
\text { resolving }\end{array}$ \\
\hline 5 & Right MCA & TCD day 4 & $\begin{array}{l}\text { Cortical, pronounced parasagittal and } \\
\text { moderate right MCA territory changes }\end{array}$ & None & Increased lactate & Increased \\
\hline 6 & Left MCA and ACA & DSA day 3 & Cortical, moderate, left MCA territory & None & Not performed & NS \\
\hline 7 & Right MCA & DSA day 7 & $\begin{array}{l}\text { Cortical, pronounced, right } \mathrm{MCA} \\
\text { territory }\end{array}$ & None & Decreased NAA & NS \\
\hline 8 & Left MCA and ACA & DSA day 12 & Cortical, moderate, left MCA territory & None & Normal & Increased \\
\hline 9 & None & DSA day 11 & Cortical, pronounced, left temporal lobe & None & Increased lactate & Increased \\
\hline 2nd study & & & Unchanged & None & Normal & $\begin{array}{l}\text { Increase } \\
\text { resolving }\end{array}$ \\
\hline 10 & $\begin{array}{l}\text { Presumed: clinical deterioration } \\
\text { with hypodense areas on CT }\end{array}$ & day 8 & $\begin{array}{l}\text { Cortical, pronounced right frontal and } \\
\text { moderate left MCA territory changes }\end{array}$ & Pronounced & Normal & NS \\
\hline 11 & Right MCA and bilateral ACA & TCD day 10 & $\begin{array}{l}\text { Cortical, pronounced parasagittal and } \\
\text { moderate Rt MCA territory changes }\end{array}$ & None & Increased lactate & Decrease \\
\hline 2nd study & & & Partial resolution & Moderate & Lactate resolving & $\begin{array}{l}\text { Decrease } \\
\text { resolving }\end{array}$ \\
\hline
\end{tabular}

$\mathrm{ACA}=$ anterior cerebral artery; $\mathrm{MCA}=$ middle cerebral artery; $\mathrm{DSA}=$ digital subtraction angiogram; $\mathrm{TCD}=$ transcranial $\mathrm{Doppler}$ ultrasonography; $\mathrm{CT}=\mathrm{computed}$ tomography; $\mathrm{MRS}=$ proton magnetic resonance spectroscopy; NAA=N-acetyl aspartate; NS refers to a non-significant asymmetry in relative cerebral blood volume (CBV). 

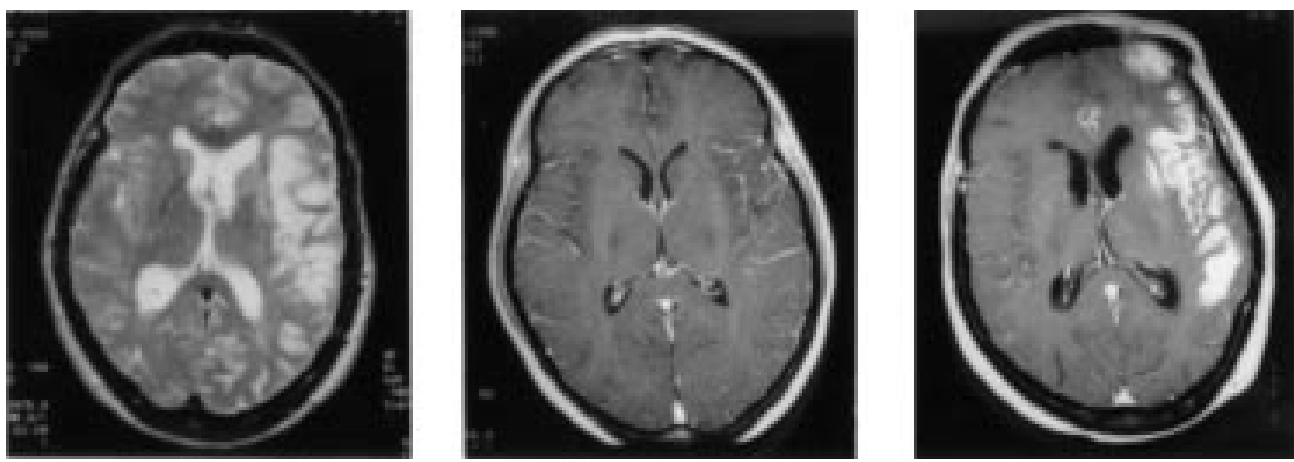

Figure 1 Typical MRI changes. Left: T2 weighted image showing oedema with sulcal effacement and high signal in the left middle cerebral artery territory. Centre: T1 weighted image after gadolinium, without parenchymal enhancement. Right: parenchymal enhancement with gadolinium was seen, but only late, a minimum of 14 days after subarachnoid haemorrhage.

of $0.5 \mathrm{mM}$ gadolinium DTPA were given intravenously, starting on the 10th image, through a 20 gauge antecubital line, or a central line if present. T1 weighted images (spin echo sequence, TE $14 \mathrm{~ms}$, TR $651 \mathrm{~ms}$ ) were then acquired to assess parenchymal enhancement with gadolinium, indicative of a breakdown of the blood brain barrier. Localised proton spectroscopy was performed with a PRESS spectroscopic imaging technique. ${ }^{12}$ The PRESS sequence (TE $135 \mathrm{~ms}$, TR $1.5 \mathrm{~s}$ ) selected signal from a $50 \times 50 \times 15 \mathrm{~mm}^{3}$ volume extending from the region of interest into the surrounding brain. Signal from within this volume was further localised using spectroscopic imaging ( $16 \times 16$ phase encodes) yielding nominal voxels of $10 \times 10 \times 15 \mathrm{~mm}^{3}$. The region of interest was selected to include the oedematous area on the $\mathrm{T} 2$ weighted image.

The total mean time in the magnet for patients completing the protocol was 42 (SD 6) minutes. This was achieved by using the standard clinical imaging sequences and limiting the spectroscopy preparation time by utilising the autoshim. The time taken for each stage of the protocol was as follows: scouts 30 seconds, T2 weighted MRI two minutes, DPS preparation 36 seconds, DPS acquisition 96 seconds, T1

A

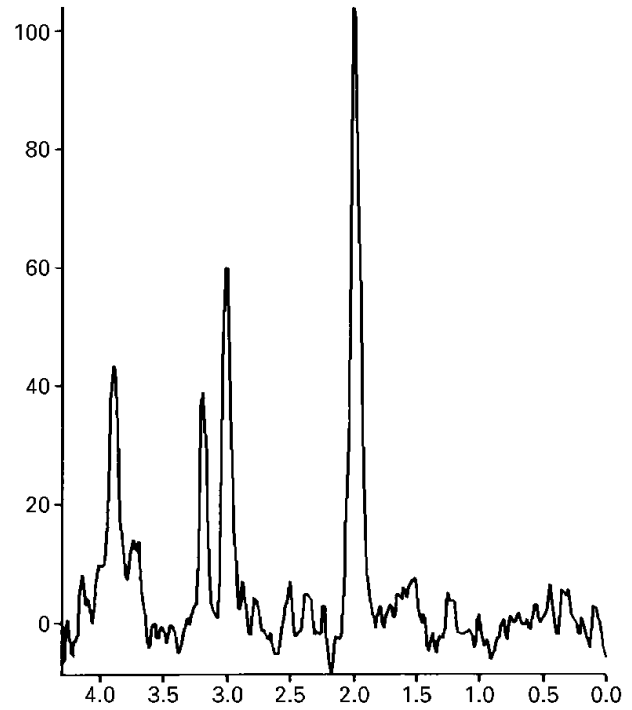

weighted MRI four minutes, MRS preparation about 10 minutes, MRS acquisition $13 \mathrm{~min}$ utes. Non-invasive blood pressure, pulse oximetry, and electrocardiographic monitoring were available for all patients.

\section{DATA ANALYSIS:}

The T1 and T2 weighted images were reported blindly and independently by consultant neuroradiologists. Signal changes suggestive of oedema and parenchymal enhancement with gadolinium were graded as absent (0), moderate (1), or pronounced (2).

Spectroscopy data were analysed using the Siemen's software which displayed spectra from $50 \times 50 \times 15 \mathrm{~mm}^{3}$ slice as contiguous $10 \times 10$ $\mathrm{mm}^{2}$ voxels. Spectral peaks from creatine $(\mathrm{Cr})$, choline (Ch), N-acetyl aspartate (NAA), and lactate if present, were fitted to Lorentzian line shapes and integrated. Line widths were used to assess paramagnetic effects. The combined value of the creatine and choline integrals $(\mathrm{Cr}+\mathrm{Ch})$ was used as a reference and the other metabolite integrals expressed as ratios relative to this in addition to using $\mathrm{Cr} / \mathrm{Ch}$ as a constant. Where adjacent voxels showed the same qualitative changes, the ratios were averaged together to give a mean value for that region. The

B

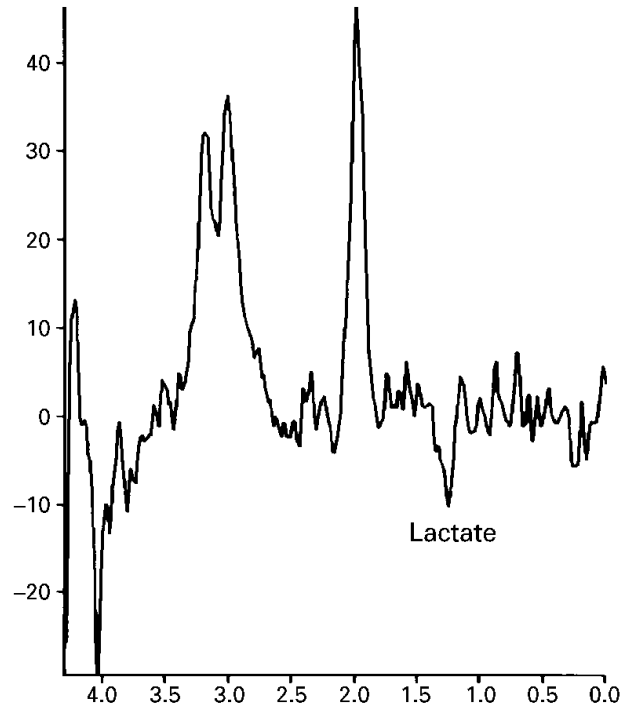

Figure 2 Illustrative spectra (A) from a good grade patient with normal metabolite ratios and (B) from a poor grade patient showing reduced NAA and a lactate peak. 
Table 3 Mean (SD) values for the metabolite ratios, and for the percentage asymmetry in relative cerebral blood volume related to $(A)$ the Hunt and Hess clinical grade, $(B)$ to the degree of oedema assessed on the $T 2$ weighted image, and $(C)$ individual values generated from follow up studies

(A) Changes with Hunt and Hess grade

\begin{tabular}{|c|c|c|c|}
\hline Clinical grade & 1 and 2 & 3 & 4 \\
\hline $\begin{array}{l}\text { Lactate } /(\mathrm{Ch}+\mathrm{Cr}) \\
\mathrm{NAA} /(\mathrm{Ch}+\mathrm{Cr}) \\
\mathrm{Cr} / \mathrm{Ch} \\
\% \text { relative } \mathrm{CBV} \\
\text { (B) Changes with oedemo }\end{array}$ & $\begin{array}{l}0.0(0.0) \\
0.84(0.22) \\
1.34(0.26) \\
-8(23) \%\end{array}$ & $\begin{array}{l}0.09(0.10) \\
0.74(0.18) \\
1.03(0.19) \\
52(47) \%\end{array}$ & $\begin{array}{l}0.41(0.58) \\
0.29(0.08) \\
1.02(0.16) \\
79(94) \%\end{array}$ \\
\hline Oedema & Absent & Moderate & Pronounced \\
\hline $\begin{array}{l}\text { Lactate/(Ch+Cr) } \\
\mathrm{NAA} /(\mathrm{Cr}+\mathrm{Ch}) \\
\mathrm{Cr} / \mathrm{Ch}\end{array}$ & $\begin{array}{l}0.01(0.02) \\
0.83(0.18) \\
1.09(0.23)\end{array}$ & $\begin{array}{l}0.01(0.02) \\
0.89(0.24) \\
1.23(0.26)\end{array}$ & $\begin{array}{l}0.16(0.24) \\
0.63(0.23) \\
0.99(0.16)\end{array}$ \\
\hline \multicolumn{4}{|c|}{ (C) Improved metabolite ratios on repeat studies (three patients) } \\
\hline & Initial study & & Follow up study \\
\hline $\begin{array}{l}\text { Time from SAH (days) } \\
\text { Lactate/(Ch+Cr) } \\
\text { NAA/(Cr+Ch) } \\
\mathrm{Cr} / \mathrm{Ch}\end{array}$ & $\begin{array}{l}13,13,12 \\
0.25,0.62,0.82 \\
0.78,0.62,0.29 \\
1.02,0.91,1.10\end{array}$ & & $\begin{array}{l}19,27,15 \\
0,0,0 \\
0.81,0.83,0.44 \\
1.00,1.12,1.02\end{array}$ \\
\hline
\end{tabular}

$\mathrm{Ch}=$ choline $\mathrm{Cr}=$ creatine $\mathrm{NAA}=\mathrm{N}=$ acetyl aspartate $\mathrm{CBV}=$ cerebral blood volume; $\mathrm{SAH}=$ subarachnoid haemorrhage. out vasospasm, one (case 2) was studied on day 3 , when she was asymptomatic and before it might have developed. A second patient (case 10) deteriorated neurologically eight days after her presenting haemorrhage, developing at that time areas of radiolucency on CT. The MR study was performed late (day 26), once her clinical condition had stabilised and the appearance of her CT resolved. The third patient (case 9), despite developing DIND nine days after the presenting subarachnoid haemorrhage, had no evidence of lumenal narrowing on concurrent angiography, and no other causative factor could be identified to account for her neurological deterioration.

\section{MRI FINDINGS}

Figure 1 shows typical images acquired in this study. All 11 patients had evidence of oedema on the $\mathrm{T} 2$ weighted scans, with increased signal intensity and effacement of the sulci. These changes were localised, were most obvious cortically, and their distribution was in keeping with the presence of focal neurological deficits. Pronounced changes of oedema were significantly more common in poor grade (3 and 4) compared with good grade patients $(p<0.01)$. Breakdown of the blood brain barrier resulting in parenchymal enhancement with gadolinium was seen in four patients, and occurred in areas where pronounced oedema had been noted on the T2 weighted scans. It seemed to be a delayed phenomenon. converted into a relative gadolinium concentration-time curve, the integral of which was used to calculate a relative cerebral blood volume map. ${ }^{13}{ }^{14}$ Changes in relative blood volume were expressed as a percentage of a symmetrically placed region of interest in the contralateral hemisphere which had a normal MR appearance. Diffuse vascular changes after subarachnoid haemorrhage affecting both hemispheres may not be detected by this method. To verify the significance of any asymmetry found, a multistage process described in the appendix was applied. In addition, the time course of the signal intensity changes were plotted to follow the passage of gadolinium through the brain, in those selected regions of interest.

Statistical comparisons used Student's $t$ test. Hunt and Hess clinical grades were compared with the severity of oedema, metabolite ratios, and changes in relative blood volume in that region. Blood volumes from distinct regions corresponding to spectroscopy voxels were used to correlate the results of the perfusion scan with the metabolite ratios. Values are quoted as mean (SD).

\section{Results}

Table 2 shows the results for the individual patients, including details of the relevant clinical investigations performed. Vasospasm was confirmed in eight patients angiographically or with transcranial Doppler within a mean of two days from the first MR study. In general the pattern of vasospasm reflected the site of the aneurysm, the location and extent of subarachnoid blood detected by CT, and concurred with the distribution of focal neurological deficits found clinically. Of the three patients with-

\section{MRS FINDINGS}

Spectra from good grade patients were normal with no lactate present (fig 2 A). Small lactate peaks were detected in three of the four initial studies carried out on grade 3 patients (fig 2 B). In the two grade 4 patients studied, both had a considerable decrease in NAA, and lactate was seen in one. In all patients, the average metabolite ratios from the most severely affected ROI correlated with the clinical grade (table $3 \mathrm{~A})$. The $\mathrm{NAA} /(\mathrm{Cr}+\mathrm{Ch})$ ratio was significantly lower in grade 4 compared with grade 3 patients $(\mathrm{p}<0.05)$, and grade 3 patients tended to have lower values than grades 1 and 2 . Conversely, the lactate/ $(\mathrm{Cr}+\mathrm{Ch})$ ratio increased with worsening clinical grade. Metabolite ratios were also related to the severity of oedema on the T2 weighted images (table $3 \mathrm{~B}$ ). Spectra from the most swollen areas of the brain had significantly lower NAA $/(\mathrm{Cr}+\mathrm{Ch})$ ratios than spectra from moderately swollen or unaffected areas $(\mathrm{p}<0.05)$. The lactate/ $(\mathrm{Cr}+\mathrm{Ch})$ ratio also tended to be higher where the oedema was most pronounced, although this did not reach significance. Changes in metabolite ratios seemed to be due to altered NAA and lactate concentrations as there were no significant changes in the $\mathrm{Cr} / \mathrm{Ch}$ ratio with changes in clinical grade or severity of oedema.

Repeat spectroscopy studies were performed in three patients. NAA $/(\mathrm{Ch}+\mathrm{Cr})$ improved in these patients, and the lactate peaks resolved (table $3 \mathrm{C}$ ). These changes accompanied clinical improvement. 

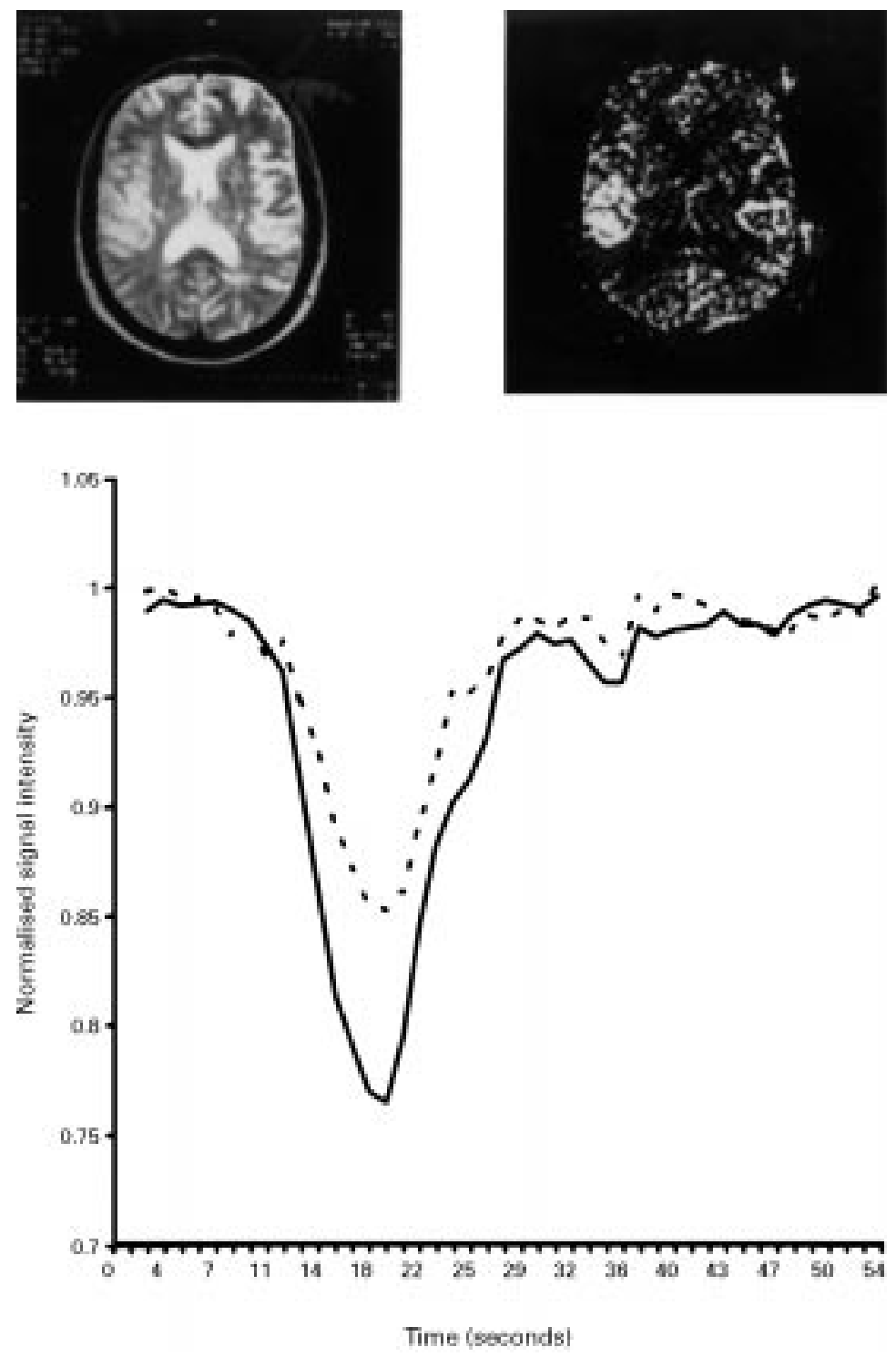

Figure 3 The relation of perfusion changes to cortical oedema. Left: T2 weighted image with oedema in the right middle cerebral artery territory. Right: increased relative blood volume (high signal) mapping to the oedematous region. Below: the time course of the signal intensity changes for the swollen high volume region (solid line), and for a symmetric region from the left (normal) hemisphere (broken line). The difference in relative blood volume is generated by the difference in amplitude, whereas the similar time course suggests that perfusion is greater in the swollen brain than in the contralateral asymptomatic side. Despite this lactate was seen in these areas of high relative cerebral blood volume.

CEREBRAL BLOOD VOLUME MEASUREMENTS

Figure 3 shows the time course of the signal changes due to the passage of gadolinium, and the cerebral blood volume map calculated from this data. Within the sensitivity of this measurement there is no difference in the temporal characteristics between the affected and contralateral hemisphere. Blood volume changes, relative to symmetric regions of interest in the contralateral hemisphere, were variable. However, if only the most swollen region of the brain defined from the T2 weighted image was considered, blood volume increased as clinical grade worsened (table 3 A). Relative blood volumes were significantly higher in grade 3 and 4 compared with grade 1 and 2 patients $(\mathrm{p}<0.05)$ and the highest blood volumes were seen in grade 4 patients.
The finding of both raised relative blood volume and lactate in regions of cerebral oedema in poor grade patients, means that lactate is associated with areas of high relative blood volume.

\section{Discussion}

There have been few MR studies of subarachnoid haemorrhage, either with conventional imaging or with spectroscopy. The advantage of a combined approach is that it provides structural, metabolic, and haemodynamic information as a single study, and so offers a potent way of exploring physiological and pathological processes. When applied to the DIND which may complicate subarachnoid haemorrhage, there are discrepancies between cerebral perfusion and metabolism, suggesting that symptomatic changes seen in DIND may not simply be the result of a reduction in blood flow.

Previous imaging studies have considered the feasibility, safety, and diagnostic use of MRI in acute subarachnoid haemorrhage. ${ }^{15-17}$ Although these series have commented on the sensitivity of MRI for detecting oedema, such changes have not been detected in preoperative studies. Postoperative changes, either at the site of the craniotomy possibly due to local trauma ${ }^{15}$ or remote from it in the deep tissues ${ }^{16}$ were described. In keeping with this similar changes were seen in the three surgically treated patients in this series. Whereas in these cases the cortical signal changes could be due to surgical trauma, similar changes were also found in the seven patients treated endovascularly and in the patient with an untreated aneurysm. Furthermore, the anatomical distribution of these changes were in keeping with the patients' focal neurological deficits, and the severity of the changes were significantly related to clinical grade. These changes may not have been noted in earlier studies because these have examined predominantly good grade patients, by contrast with the grade 3 and 4 cases in this series. The predominanently cortical distribution of these signal changes was striking, which may reflect the higher metabolic rate of grey compared with white matter, or the importance of exposure to subarachnoid blood clot and its degradation products.

Although MRS studies of subarachnoid haemorrhage are limited, the metabolic changes of cerebral ischaemia have been well documented both in animal models and in human stroke with proton and phosphorus spectroscopy. ${ }^{18}{ }^{19}$ With ischaemia and infarction, lactate accumulates and NAA is lost. Whereas acutely the lactate may reflect anaerobic metabolism, it can persist for weeks or even months after the stroke, ${ }^{20}$ when its presence has been attributed to macrophage invasion and gliosis. ${ }^{21}$ NAA is regarded as a neuronal marker, and decreases have been interpreted as a loss of neurons.

Phosphorus spectroscopy after subarachnoid haemorrhage has detected acidosis and a loss of high energy phosphates in symptomatic patients, these changes resolving with neurological recovery. ${ }^{22}{ }^{23}$ Similar changes have 
been reported in a primate model. ${ }^{24}$ The finding in this study of increased lactate and decreased NAA is therefore not surprising. The relation of the spectral changes to the clinical grade of the patients, to the severity of the oedema, and the improvement in metabolite ratios seen as patients recovered, illustrate the close association of metabolism with cellular function and the accumulation of water. ${ }^{1825}$

In this study a significant increase in the relative blood volume was seen in the most swollen regions of the brain of patients in the poorest clinical grades. Grubb et al, in an early study using fixed iodide counters, reported a $58 \%$ increase in blood volume in 14 examinations of poor grade patients with angiographic lumenal narrowing, but variable changes in a further 31 examinations of patients without vasospasm or in better grades. ${ }^{4}$ Subsequent PET studies have confirmed the finding of increased blood volume,${ }^{78}$ or have reported no change. ${ }^{56}$ This increase in blood volume in poor grade patients may represent dilatation of the intraparenchymal vessels compensating for proximal vasospasm. ${ }^{4}$

This study has shown the practicality of using an integrated MR protocol in patients with subarachnoid haemorrhage to investigate structure, metabolism, and cerebral blood volume. The results have confirmed a close relation between lactate, oedema, and clinical grade. In keeping with previous studies, increased relative cerebral blood volume has also been associated with these changes. Reflecting these associations lactate is found in areas of high relative cerebral blood volume. The understanding of this relation and the determination of parenchymal perfusion and metabolism in these areas may alter the approach to patient management.

\section{Appendix}

To test whether asymmetries in relative blood volume measurements were significant, the following process was applied:

(1) The relative gadolinium concentrationtime curve $[\mathrm{C}(\mathrm{t})]$ for the region of interest, and for a symmetric region of interest from the contralateral hemisphere were determined. Relative blood volume is the integral of this curve.

(2) One curve was subtracted from the other to generate a relative concentration difference time course $[\Delta \mathrm{C}(\mathrm{t})]$.

(3) The variance $\left[\sigma^{2}\right]$ in this concentration difference-time course due to noise was calculated from the points before the signal change due to the gadolinium.

(4) The time course of the $\mathrm{z}$ score $[\mathrm{z}(\mathrm{t})]$ was generated by dividing the concentration differences by the standard deviation calculated from the noise level:

$\mathrm{z}(\mathrm{t})=\Delta \mathrm{C}(\mathrm{t}) / \sigma$

(5) The concentration-time curves were then defined as differing significantly when $\mathrm{z}(\mathrm{t})$ averaged over three consecutive points exceeded $1.96(\mathrm{p}<0.05)$ during the passage of the gadolinium.

1 Kassell NF, Torner JC, Jane JA, Haley EC, Adams HP. The international cooperative study on the timing of aneurysm surgery : part 1. f Neurosurg 1990;73:18-36.

2 Pickard JD, Nelson R, Martin JL. Pathophysiology of aneurysmal subarachnoid haemorrhage. In: Teasdale G, Miller J, ed. Current neurosurgery. Edinburgh: Churchill Livingstone, 1992:1-38.

3 Sloan MA. Detection of vasospasm following subarachnoid haemorrhage. In: Babikian VL, Wechsler LR, eds. Transcranial Doppler ultrasonography. St Louis: Mosby, 1993;105-27

4 Grubb R, Raichle M, Eichling J, Gadd M. Effects of subarachnoid hemorrhage on cerebral blood volume, blood flow and oxygen utilisation in humans. $\mathcal{f}$ Neurosurg 1977;46:446-53.

5 Carpenter D, Grubb R, Tempel L, Powers W. Cerebral oxygen metabolism after aneurysmal subarachnoid haemorhage. F Cereb Blood Flow Metab 1991;11:837-44

6 Powers W, Grubb R, Baker R, Mintun M, Raichle M. Regional cerebral blood flow and metabolism in reversible ischemia due to vasospasm. $\mathcal{F}$ Neurosurg 1985;62:539-46.

7 Hino A, Mizukawa N, Tensin H, et al. Postoperative hemodynamic and metabolic changes in patients with subarachnoid hemorrhage. Stroke 1989;20:1504-10.

8 Kawamura S, Sayama I, Yasui N, Uemura K. Sequential changes in cerebral blood flow and metabolism in patients with subarachnoid haemorrhage. Acta Neurochir (Wien) 1992;114:12-5

9 Martin W, Baker R, Grubb R, Raichle M. Cerebral blood volume, blood flow and oxygen metabolism in cerebral ischaemia and subarachnoid haemorrhage: an in vivo study using positron emission tomography. Acta Neurochir (Wien) 1984;70:3-9.

10 Hunt W, Hess R. Surgical risk as related to time of intervention in the repair of intracranial aneurysms. $\mathcal{f}$ Neurosurg 1968;28:14-20.

11 Fisher C, Kistler J, Davis J. Relation of cerebral vasospasm to subarachnoid hemorrhage visualised by computerised tomographic scanning. Neurosurgery 1980;6:1-9.

12 Bottomley PA. Spatial localization in NMR spectroscopy in vivo. Ann N Y Acad Sci 1987;508:333-48.

13 Robson M, Zhong J, Gore J. Errors associated with fitting dynamic susceptibility contrast images for cerebral perdynamic susceptibility contrast images for cerebral per-
fusion calculations. 3rd Proceedings of the Society of Magnetic fusion calculations. 3rd Proceedings of the Society of Magnetic
Resonance and 12th Meeting of the European Society for MagResonance and 12th Meeting of the European Society for Mag-
netic Resonance in Medicine and Biology. Nice, France: 1995: 869.

14 Rosen DR, Belliveau JW, Vevea JM, Brady TJ. Perfusion imaging with NMR contrast agents. Magn Reson Med 1990;14:249-65.

15 Jenkins A, Hadley DM, Teasdale GM, Condon B, Macpherson P, Patterson J. Magnetic resonance imaging of acute subarachnoid hemorrhage. F Neurosurg 1988;68:731-6.

16 Matsumura K, Matsuda M, Handa J, Todo G. Magnetic resonance imaging with aneurysmal subarachnoid haemorrhage: comparison with computed tomography scan. Surg Neurol 1990;34:71-8.

17 Satoh S, Kadoya S. Magnetic resonance imaging of subarachnoid haemorrhage. Neuroradiology 1988;30:361-6.

18 Gadian D, Williams S, Bates T, Kauppinen R. NMR spectroscopy: current status and future possibilities. Acta Neurochir Suppl (Wien) 1993;57:1-8.

19 Welch K, Levine S, Martin G, Ordidge R, Vande-Linde AM, Helpern JA. Magnetic resonance spectroscopy in cerebral ischemia. Neurol Clin 1992;10:1-29.

20 Graham GD, Blamire AM, Rothman DL, et al. Early temporal variations of cerebral metabolites after human stroke. A proton magnetic resonance spectroscopy study. Stroke 1993;24:1891-6

21 Petroff O, Graham G, Blamire A, et al. Spectroscopic imaging of stroke in humans: histopathology correlates of spectral changes. Neurology 1992;42:1349-54.

22 Brooke N, Ouwerkerk R, Adams CBT, Radda GK, Ledingham JGG, Rajagopalan B. Phosphorus-31 magnetic resonance spectra reveal prolonged intracellular acidosis in the brain following subarachnoid haemorrhage. Proc Natl Acad Sci USA 1994;91:1903-7.

23 Rowe JG, Soper N, Ouwerkerk R, Kerr RS, Radda GK, Rajagopalan B. Delayed ischaemia after subarachnoid haemorrhage: a role for small vessel changes. $f$ Neurol Neurosurg Psychiatry 1995;59:451-2.

24 Handa Y, Kubota T, Tsuchida A, et al. Effect of systemic hypotension on cerebral energy metabolism during chronic cerebral vasospasm in primates. $\mathcal{f}$ Neurosurg 1993;78:112-9.

25 Siesjo B. Pathophysiology and treatment of focal cerebral ischaemia: part 1 pathophysiology. $\mathcal{F}$ Neurosurg 1992;77: $169-84$ 